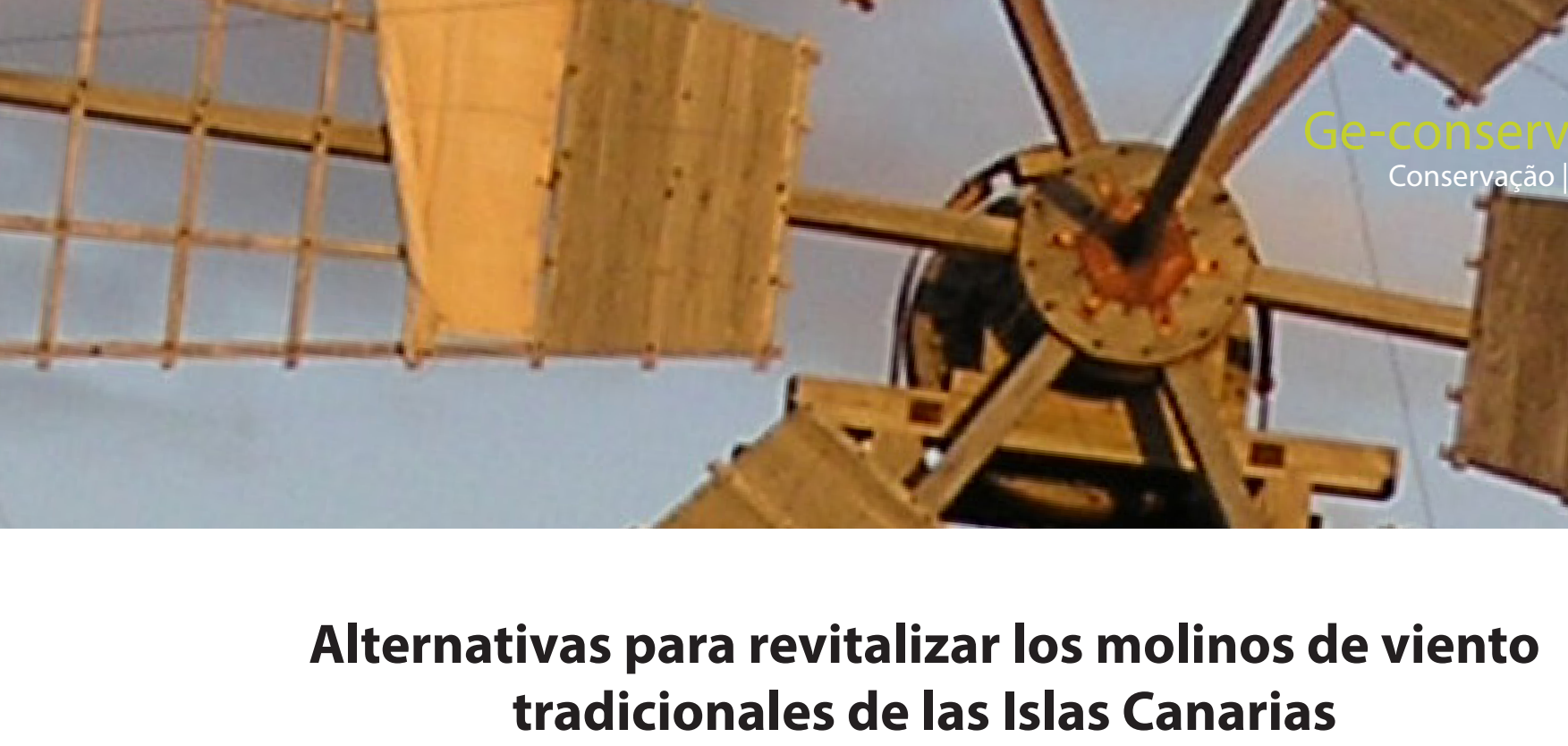

\title{
Alternativas para revitalizar los molinos de viento tradicionales de las Islas Canarias
}

\author{
Víctor Manuel Cabrera García
}

Resumen: Los molinos de viento tradicionales son unas construcciones singulares de la arquitectura tradicional que pertenecen al Patrimonio Industrial y que no son útiles para la sociedad actual ya que han desaparecido los modos de vida a los que iban ligados, por lo que se facilita el abandono y la no conservación de los mismos. Como método alternativo de conservación se propone recuperar el funcionamiento de estos molinos de viento, dotándoles de un nuevo uso que consiste en producir energía eléctrica a través de la energía renovable del viento. Esta iniciativa posibilitaría recuperar y conservar lo que aún no se perdido de estas construcciones procedentes de la cultura industrial tradicional canaria.

Palabras clave: molinos tradicionales, conservación, rehabitalización, revitalización, patrimonio industrial

\section{Alternatives to revitalize the traditional windmills of the Canary Islands}

Abstract: The traditional windmills are unique constructions of the traditional architecture that belong to the Industrial Patrimony and that are not useful for the present society since they have disappeared the ways of life to which they were tied, reason why it facilitates the abandonment and the not conservation of the same. As an alternative method of conservation, it is proposed to restore the operation of these windmills by providing them with a new use consisting of producing electrical energy through the renewable energy of the wind. This initiative would make it possible to recover and preserve what has not yet been lost from these constructions from the traditional Canarian industrial culture.

Keyword: traditional mills, conservation, rehabitalization, revitalization, industrial heritage

\section{Alternativas para revitalizar os moinhos de vento tradicionais das Ilhas Canárias}

Resumo: Os tradicionais moinhos de vento são construções singulares da arquitetura tradicional que pertencem ao Património Industrial e que não são úteis para a sociedade atual, uma vez que os modos de vida a que estavam ligados desapareceram, o que facilita o abandono e a não conservação dos mesmos. Como método alternativo de conservação, propõe-se recuperar o funcionamento destes moinhos de vento, dotando-os de um novo uso que consiste em produzir energia elétrica através da energia renovável do vento. Esta iniciativa permitiria recuperar e conservar o que ainda não foi perdido destas construções provenientes da cultura industrial tradicional das Canárias.

Palavras-chave: moinhos tradicionais, conservação, reabilitação, revitalização, património industrial 


\section{Introducción}

Durante el transcurso de los años se ha observado el progresivo abandono en muchas construcciones que pertenecen a la arquitectura tradicional canaria y que en ocasiones, no están incluidos como bienes en la Ley de Patrimonio Histórico Canario. La desaparición de los modos de vida a lo que estas edificaciones iban ligados, así como la homogenización de la cultura y la globalización socio-económica hacen que la gran variedad de los bienes materiales e inmateriales procedentes de la sociedad tradicional canaria se encuentran en situación de extrema vulnerabilidad y presentan problemas de obsolescencia e integración en la sociedad actual.

Hace varias décadas que la vida en las áreas rurales giraba en torno a las labores desarrolladas en el campo, lo que supuso crear mediante un lento proceso histórico una riqueza paisajística y patrimonial que se refleja en sus edificaciones. Sin embargo, con la llegada del turismo como nueva actividad económica a las islas Canarias en primer lugar así como la introducción de nuevas máquinas en segundo lugar, propiciaron que los municipios de carácter agrícola sufrieran un fuerte retroceso, lo que supuso que sus campos se abandonaran y sus habitantes se marcharon a los centros urbanos en busca de nuevas oportunidades laborables y de vida. Estos procesos migratorios originan transformaciones en el medio físico rural y urbano así como la pérdida de numerosos oficios favorecieron que las edificaciones quedaran obsoletas al caer en desuso.

La Comunidad Autónoma de Canarias elaboró La Ley 4/1999, del 15 de marzo del Patrimonio Histórico de Canarias que posteriormente es modificada por la Ley 11/2002, del 21 de noviembre, donde en el Titulo Preliminar de La Ley se define: "La finalidad de la presente Ley es la protección, conservación, restauración, acrecentamiento, investigación, difusión, fomento y transmisión en las mejores condiciones posibles a las generaciones futuras del patrimonio histórico de Canarias, así como su disfrute por los ciudadanos como objeto cultural y educativo y de su aprovechamiento como recurso económico, en tanto los usos armonicen con la referida finalidad".

Los molinos de viento son un tipo de edificaciones singulares de la arquitectura tradicional que quedaron obsoletas al caer en desuso y por tanto, han pasado al olvido. Son unas construcciones preindustriales que están condicionadas por las singularidades climáticas de la zona en la que se enclavany, al mismo tiempo, por el empleo de los materiales constructivos locales, adaptadas tanto al medio físico en el que se ubican como a la actividad desarrollada por sus habitantes. Los molinos de viento tradicionales son máquinas eólicas lentas cuyo funcionamiento se basa en el empuje que ejerce el viento sobre un rotor de aspas o de velas y que permiten transformar la energía cinética en energía mecánica, a través de la cual, se acciona la maquinaria principalmente para la molienda de los cereales (Rojas; Gómez; Castro 2013)
A pesar de los diversos indicadores de protección que establece la Ley del Patrimonio Histórico de Canarias, los molinos de viento tradicionales no están lo suficientemente protegidos por la legislación, ya que tan solo unos pocos se han incoado y declarado como Bien de Interés Cultural y un gran número de ellos se encuentran abandonados y en avanzado estado de ruinas al borde de la desaparición. De las numerosas entrevistas realizadas a distintos propietarios de estos inmuebles ante la pregunta de por que se encuentran estos edificios en estado de abandono, en la totalidad de los casos se afirma que no pueden hacerse cargo de la reparación de los mismos por el alto coste económico que conlleva, a pesar de que por Ley están obligados a conservarlos. Cabe señalar que la mayoría de los tradicionales molinos de viento no están incluidos como bienes en el Patrimonio Histórico de Canarias, con lo que las administraciones competentes no garantizan el mantenimiento y su conservación, con independencia de su titularidad jurídica.

Los molinos de viento tradicionales son un legado que hemos recibido de nuestros antecesores y que forman parte de nuestra historia e identidad, por lo que debemos realizar entre todos los ciudadanos, las administraciones públicas y las entidades privadas, un gran esfuerzo para conocer, recuperar y conservar lo que aún no se perdido de estas construcciones singulares de la arquitectura tradicional y del Patrimonio Industrial. No es cuestión por tanto de adoptar una postura nostálgica respecto a estos inmuebles, sino todo lo contrario, ya que estos inmuebles son máquinas eólicas construidas para desarrollar un trabajo en concreto, por lo que son algo más que un mero recuerdo sentimental de un pasado reciente.

Esas construcciones singulares son unos ingenios artesanales que sirven de testigo de como se desarrolló una parte de la cultura agrícola e industrial tradicional de Canarias en tiempos pasados y los que han sobrevivido al paso del tiempo no sólo ofrecen datos sobre la economía y técnicas artesanales que se usaban en épocas anteriores, sino también sobre la capacidad de los habitantes canarios para aprovechar los materiales disponibles en el entorno insular. La desaparición de los modos de vida a los que estos molinos de viento iban ligados así como la fragilidad de sus construcciones relacionados con el envejecimiento de los materiales de construcción frente a las inclemencias climáticas (viento, sol y lluvia) y el ausente mantenimiento de los mismos durante décadas debido a la falta de uso, los hacen especialmente vulnerables ante el abandono y el desinterés sistemático por parte de la sociedad actual, quedando en pie en muchos casos viejas estructuras y vestigios de lo que fueron en épocas pasadas.

En las islas de Fuerteventura y Lanzarote se llegaron a plantear diversas estrategias para la recuperación y restauración de los molinos de viento tradicionales. En la isla de Fuerteventura, en junio de 1985 el Cabildo puso en marcha un programa de restauración, rehabilitación y difusión del Patrimonio Histórico, con el objetivo profundizar sobre la conservación de los bienes muebles e inmuebles y la ampliación de la oferta cultural de la isla, tanto para la población insular como 
para las personas que la visitan y que desean conocer la cultura tradicional. El Cabildo creó una red insular de Museos y Centros de Interpretación con el fin de dotar a la isla de una infraestructura museística que recoja las principales manifestaciones culturales del devenir histórico insular. Se procedió a la recuperación de 23 molinos de viento tradicionales, con el objetivo de ampliar la oferta cultural de la Isla, mediante la creación de la "ruta de los molinos" que se culminaba con la creación del Centro de Interpretación de Tiscamanita. En la isla de Lanzarote, en junio 1992 el Cabildo inició un plan de restauración de numerosos molinos de viento tradicionales con la finalidad de que se restaurasen los edificios, los rotores de aspas, los mecanismos de las maquinarias y de esta manera incorporarlos a la oferta museística de la isla, como recuerdos históricos estáticos de las antiguas técnicas artesanales, agrícolas e industriales que hubo en épocas pasadas en la isla (Cabrera 2010)

De los molinos de viento protegidos por la legislación y declarados como B.I.C en el archipiélago canario, tan solo se conservan en buen estado el molino de viento harinero"Tipo Torre" del Quemado [figura 1], en el municipio de Mogán en la isla de Gran Canaria, el molino de viento harinero "Tipo Torre" en Tiscamanita [figura 2], y el molino de viento harinero "La Molina" en Tefía [figura 3], ambos en la isla de Fuerteventura. Estos dos últimos conservan su uso original como recurso turístico, y se ponen en funcionamiento de vez en cuando con la finalidad de obtener el gofio que posteriormente se vende como un producto artesanal en las tiendas de artesanía que conviven con los molinos.

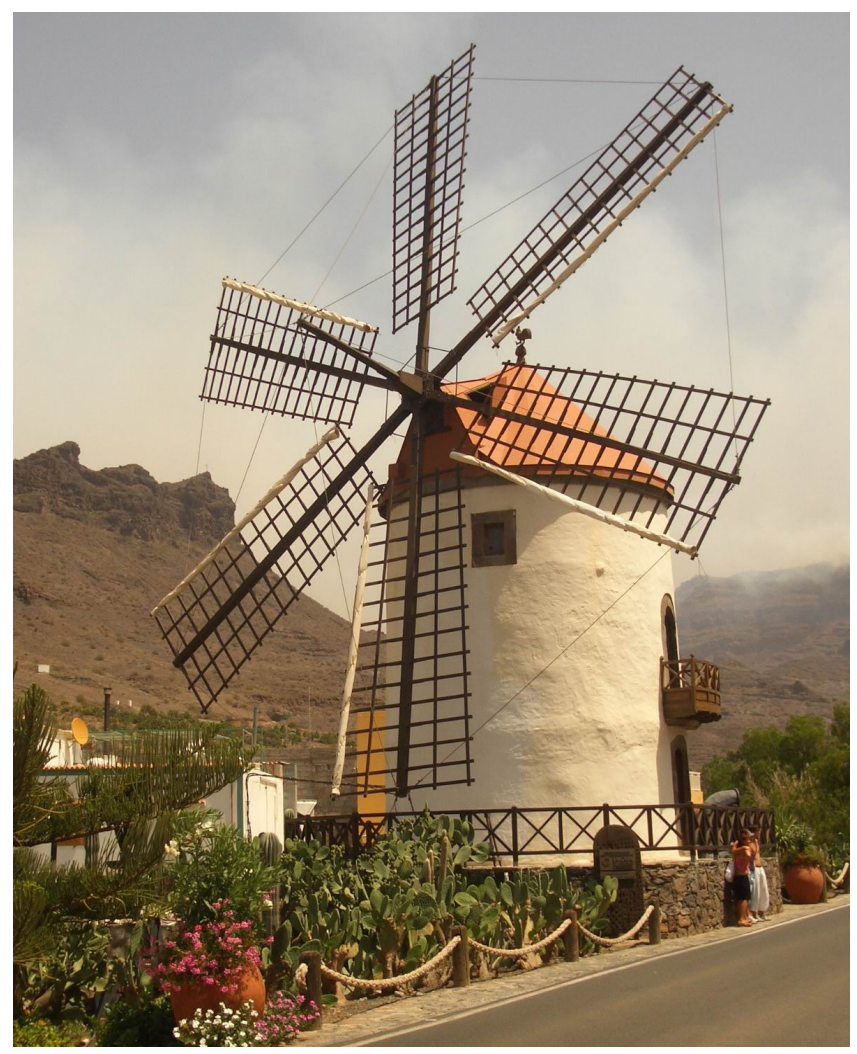

Figura 1.- Molino harinero "Tipo Torre" en TM de Mogán, Gran Canaria. Fotografía: Víctor M. Cabrera García

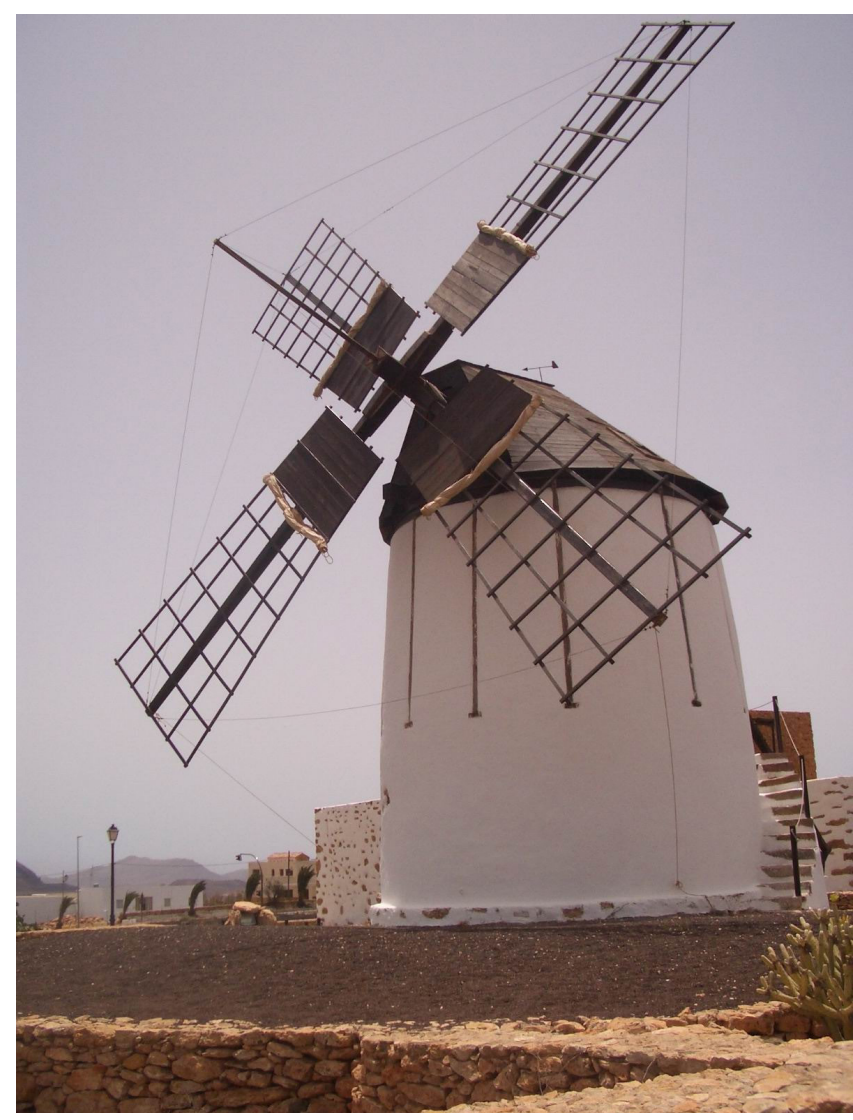

Figura 2.- Molino harinero "Tipo Torre". Tefía, TM de Puerto del Rosario Fuerteventura. Fotografía: Víctor M. Cabrera García

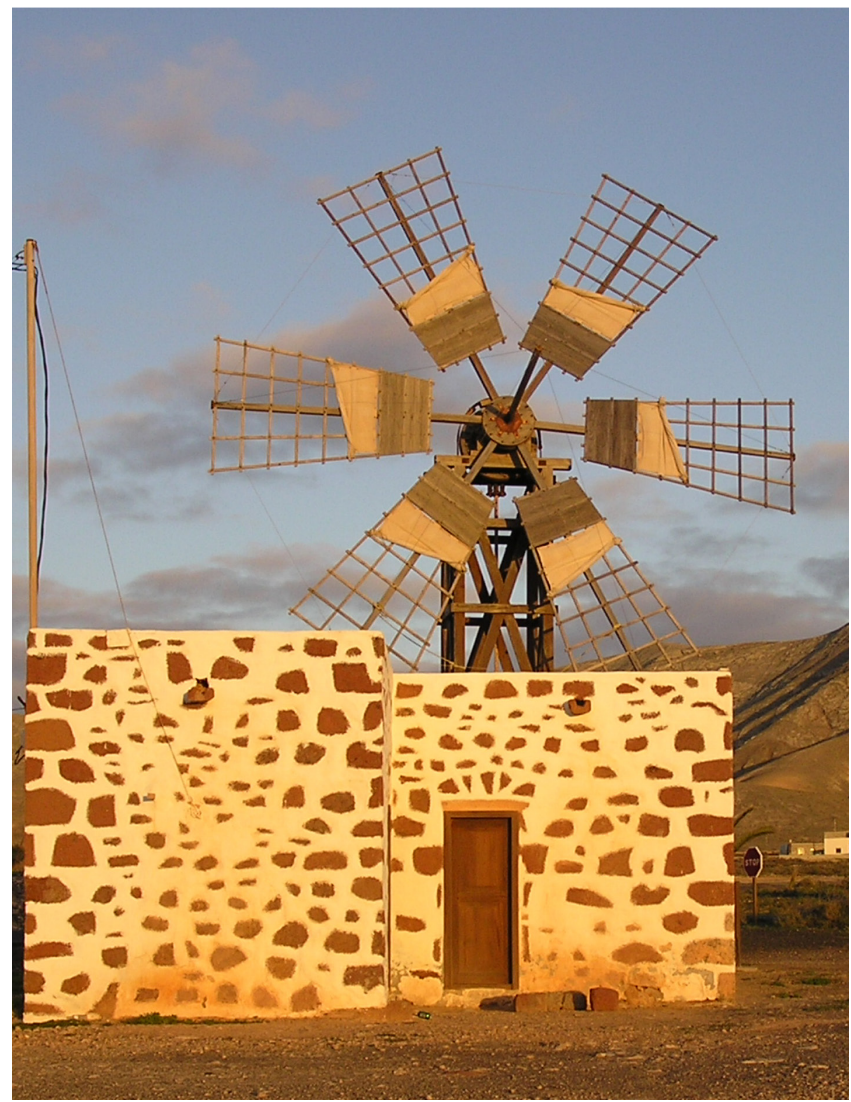

Figura 3.- Molino harinero "La Molina". Tefía, TM de Puerto del Rosario Fuerteventura. Fotografía: Víctor M. Cabrera García 
Se concluye por tanto que uno de los mejores métodos para conservar los edificios procedentes de la cultura tradicional es dotarles de uso, incluso, de proponer usos alternativos que sean compatibles con sociedad actual y que al mismo tiempo sean respetuosos con estas construcciones tradicionales. Sin embargo, gran número de los molinos de viento tradicionales inventariados en el archipiélago canario al carecer de uso presentan deterioros importantes en los elementos materiales que conforman los edificios, en los rotores de aspas y en la maquinaria, debido fundamentalmente a la escasez de mantenimiento, lo que conlleva la aparición de la erosión en los materiales constructivos propiciados por los agentes atmosféricos y por consiguiente se facilita el abandono de los mismos.

Respecto a la conservación de los bienes patrimoniales, la administración estatal ha creado una serie de instrumentos de gestión del Patrimonio denominados Planes Nacionales (Plan Nacional del Paisaje Cultural, Plan Nacional de Investigación del Patrimonio, Plan Nacional de la Conservación Preventiva, Plan Nacional de Arquitectura Tradicional y Plan Nacional del Patrimonio Industrial). Dichos planes se crean para conseguir tres fines:

- Establecer una metodología de actuación unificada sobre el conjunto de bienes.

- Programar las inversiones a realizar de acuerdo con las necesidades de conservación.

- Coordinar la participación de las distintas instituciones que intervienen en la conservación de los conjuntos patrimoniales.

La arquitectura tradicional está ligada a los modos de vida y a la organización social de un territorio, surge como respuesta a las necesidades cotidianas de sus usuarios y viene marcada por la funcionalidad, la economía, la estructura de la propiedad, las clases sociales, las creencias y simbolismo, la tradición, la historia del lugar y la zona geográfica donde se produce e inserta. Es un todo inseparable de la vida de las gentes que la protagonizan y habitan. Por tanto, el Patrimonio arquitectónico construido forma parte del Patrimonio cultural de un determinado entorno, ya que su naturaleza se basa en una serie de principios que le otorgan un valor relevante para la memoria colectiva de una determinada sociedad. Dicho Patrimonio no son solo las edificaciones singulares y los núcleos urbanos, sino también las edificaciones utilitarias o productivas, las infraestructuras, las terrazas realizadas en la orografía y otros elementos que conforman la transformación del ser humano en el paisaje ${ }^{[1]}$.

Los molinos de viento existentes en las islas Canarias son edificaciones singulares que pertenecen tanto al patrimonio arquitectónico como al patrimonio industrial, ya que estos elementos se emplearon para desarrollar actividades relacionadas con la cultura del trabajo y son por tanto elementos industriales con la categoría de bien industrial debido a que ha desaparecido el uso para los que fueron creados, pero que por su valor histórico y arquitectónico son testigos de una actividad agrícola e industrial desarrollada en tiempos pasados. Por lo tanto, son elementos dignos de conservar a pesar de la obsolescencia funcional que tienen actualmente, condicionados por la ausencia de rentabilidad económica además de un costoso mantenimiento. Los modos de intervención en el Patrimonio Industrial deben de centrar el interés no solo en los edificios sino también en los factores territoriales que condicionan la puesta en valor del espacio productivo y del paisaje donde se ubican. El Patrimonio Industrial en épocas pasadas se consideró de escaso valor intrínseco y potencial, en contraste con el Patrimonio histórico, artístico y arquitectónico, por lo tanto existen contradicciones sociales entre su conservación como elementos meramente arquitectónicos (no siempre valorados positivamente) y su costosa reutilización para la puesta en valor (Benito; Calderón; Ruiz 2016)

La solución clásica de restauración y rehabilitación consiste en crear numerosos Centros de Interpretación para garantizar la conservación de la mayor parte de las construcciones de la arquitectura tradicional como meros recuerdos históricos de las antiguas técnicas artesanales, agrícolas o industriales por su gran valor didáctico y para incentivar el estudio de la evolución de una determinada sociedad en el curso del tiempo, asumiendo la pérdida de la forma de vida a las que daban origen. Habitualmente se utilizan dos tipos estrategias, de un lado mediante la protección de las edificaciones mediante la legislación y de otro lado, revitalizar el medio rural. Sin embargo, en la actualidad estas acciones resultan del todo insuficientes para la conservación de este tipo de construcciones, ya que la mayoría de estos elementos, salvo escasas excepciones, se encuentran en un estado avanzado de ruinas y de abandono.

En España se han llevado a cabo diversas estrategias para garantizar la conservación los elementos pertenecientes al Patrimonio Industrial, una de ellas se ha desarrollado en los deteriorados recintos industriales existentes en algunas ciudades, incorporando usos más rentables como lo son los usos culturales y turísticos (PARDO 2004). La transformación de fábricas en muesos relacionados con la actividad cultural y la incorporación de los usos relacionados con el ocio suponen tener una rentabilidad económica que propicia la renovación del entorno urbano más próximo, sin embargo en ocasiones, las tensiones económicas originadas por la presión inmobiliaria en estos nuevos espacios, generan tensiones que originan, en ocasiones, la destrucción de los viejos edificios industriales como nueva estrategia para generar un nuevo ciclo de revaloración del entorno físico cercano.

Otra de las estrategias llevadas a cabo son las de recuperar y la puesta en valor de los edificios patrimoniales como recurso turístico que contribuye al desarrollo local desde la visión cultural, social y económica. El binomio patrimonio y turismo ayudará a entender la dimensión geográfica del Patrimonio Industrial, recurso capaz de regenerar entornos físicos y revalorizar el paisaje. Se reorienta el 
interés turístico no solo a los edificios aislados sino que se generan itinerarios temáticos que enlazan las edificaciones industriales dispersas que explican la actividad industrial del territorio. En este sentido existen experiencias en esta línea en Cataluña, Castilla la Mancha, Comunidad Valenciana, Asturias, Castilla León, Andalucía, Cantabria, Galicia y en Extremadura, sin embargo, Canarias no tiene apenas experiencia en este sector.

Estas acciones deben de mostrarse con fidelidad y rigor para no desvirtuar la memoria del trabajo así como la historia técnica y social del lugar. El peligro estará en plantear actuaciones de intervención-rehabilitación con planteamientos habituales de uso turístico y de desarrollo económico, olvidando la dimensión cultural que tiene el Patrimonio Industrial. El turismo industrial puede hacer viable la combinación de viejos edificios con nuevas y complejas estructuras. Las áreas con elementos del Patrimonio Industrial se pueden convertir también en unos buenos productos turísticos y generar beneficios económicos, ya que atraen a las familias y a los visitantes de cualquier nivel social y cultural.

Del mismo modo, se está viviendo un proceso de revalorización de los ámbitos rurales convirtiéndose en destinos turísticos, lo que ha conllevado un mayor desarrollo, inversión y protección del mundo rural, recuperando la importancia territorial perdida en las últimas décadas, sin obviar el elemento fundamental en este proceso, que no es sino la sostenibilidad entendida como una nueva dimensión necesaria del propio desarrollo rural. El turismo rural como actividad que dinamiza la economía local y regional fomenta la recuperación del Patrimonio Industrial lo que trae consigo beneficios culturales, sociales y económicos.

Los molinos de viento forman parte de la arquitectura tradicional y etnológica, la cual casi siempre ha estado en un segundo plano respecto al resto de las edificaciones del Patrimonio arquitectónico a pesar de su relevancia sociocultural, y al caer en desudo y deterioro, se han convertido en edificio obsoletos para la sociedad actual. que ha mostrado indeferencia y falta de sensibilidad hacia este tipo de elementos del Patrimonio Histórico. Al mismo tiempo se han convertido en hitos paisajísticos que acercan al ciudadano a la cultura de la agricultura, ya que las prácticas agrarias de antaño no se realizan en la actualidad.

Sin embargo, en los Países Bajos, los molinos de viento tuvieron varias aplicaciones, por las cuales se realizaron modificaciones en sus maquinarias para desarrollar varios usos de entre los cuales destaca el bombeo de agua para la desecación de los polders (uso que se sigue manteniendo en la actualidad), el descascarillado de cebada y del arroz, la molienda de la malta, el prensado de la aceituna para obtener aceite, la molienda de cacao, mostaza y pimienta, la trituración del yeso así como los molinos aserraderos de madera (distrito de Zaam) en las proximidades de Ámsterdam (DE DECKER 2011). En España los molinos de viento se emplearon mayoritariamente para la molienda de los cereales y en menor medida, para el bombeo del agua de las zonas inferiores a cotas superiores como es el caso de los molinos de viento salineros.

Las funciones para las que se construyeron los antiguos molinos de viento se encuentran hoy día extinguidas y por lo tanto, la rentabilidad de la actividad productiva para las que fueron creados decayó con el paso del tiempo ya que con la introducción de las nuevas y potentes máquinas al servicio de una mayor rentabilidad, obligaron a los antiguos molinos de viento a caer en desuso y en consecuencia, al olvido. El valor de estos elementos pertenecientes al Patrimonio Industrial va ligado a una larga serie de aspectos (históricos, culturales, simbólicos, paisajísticos, etc.) que los hacen hoy en día ser un relevante foco de interés por la recuperación y salvaguarda del patrimonio histórico-cultural. Son numerosas las iniciativas desarrolladas hasta la fecha que han alcanzado el objetivo de recuperar el aspecto original de las edificaciones tras su catalogación como elementos patrimoniales. Pero, en demasiadas ocasiones, la recuperación de estas construcciones se limitan a la restauración de los edificios, sin llegar a plantear una gestión eficiente de los ingresos procedentes de una actividad asociada a los elementos restaurados, que permita el mantenimiento de estos edificios y en consecuencia, el patrimonio recuperado estará destinado a deteriorarse una vez más y, muy probablemente, a desaparecer con el paso del tiempo. La puesta en valor de los molinos de viento actualmente una vez recuperados también es deficiente, puesto que en muchos casos se reduce a realizar una simple visita presencial gestionada por la oficina de turismo de la localidad y no se han desarrollado herramientas eficaces complementarias que potencien el desarrollo local donde se sitúan los molinos de viento. (Castro; Rojas; Carranza 2013)

El objetivo principal de este artículo de investigación consiste en enunciar las diferentes posibilidades de recuperación de una parte del Patrimonio Industrial en las islas Canarias (los molinos de viento tradicionales) haciendo hincapié en métodos alternativos de conservación a los ya existentes. Se propone reconvertir los molinos de viento tradicionales en aerogeneradores de baja potencia, lo que supondría introducir un nuevo uso en estas construcciones singulares, con la finalidad de fomentar una gestión alternativa en relación a la conservación de estos bienes inmuebles de la arquitectura tradicional que forman parte del Patrimonio Industrial. Se trataría por lo tanto de recuperar, incorporar, reutilizar y revitalizar los diversos molinos de viento tradicionales para la sociedad actual, que mayoritariamente se encuentran en desuso, abandonados y ocasiones en estado ruinoso, mediante la puesta en valor de los mismos, permitiéndonos legarlos con garantías a las generaciones futuras.

\section{Metodología}

Las técnicas de investigación empleadas para elaborar este estudio son la técnica documental y la técnica de campo. 
La técnica documental ha permitido la recopilación de información para enunciar las teorías que sustentan el estudio realizado buscando información en libros, en revistas y en Internet en relación a las diversas máquinas eólicas existentes en las islas Canarias. Cabe destacar que la documentación existente aborda mayoritariamente las cuestiones culturales y patrimoniales de estos inmuebles, sin embargo es casi inexistente la documentación de carácter técnico referente a estas construcciones pertenecientes a la arquitectura tradicional canaria, excepto las investigaciones realizadas por el autor y que se indican en la bibliografía de este artículo.

La técnica de campo ha permitido la observación en contacto directo con los objetos de estudio que permite confrontar la teoría con la práctica en la búsqueda de la verdad objetiva. Se utiliza una metodología inductiva/deductiva. En este caso en concreto, la inducción consistió en ir de los casos particulares a la generalización de las máquinas eólicas existentes en las islas. Se inicia por la observación de unos objetos particulares como son el estudio de unos determinados molinos de viento tradicionales con el propósito de llegar a conclusiones y premisas generales, con la intención de aportar un mayor grado de conocimiento sobre los elementos de la arquitectura del viento en el archipiélago canario. De otra parte, la deducción consistió en ir de lo general a lo particular. Este método de conocimiento se inició con la observación de las diferentes máquinas eólicas existentes en Canarias con el propósito de señalar cuales son las verdaderas particularidades de las mismas, aportando mayor conocimiento sobre los aspectos constructivos, funcionales y técnicos, empleando para ello el método analítico.

\section{Diversas alternativas para favorecer la conservación de los molinos de viento}

\section{-La protección mediante la legislación}

Según el artículo 4 del Titulo Preliminar de la Ley 4/1999 del 15 de marzo de Patrimonio Histórico de Canarias, se especifica que: "........los ciudadanos y los poderes públicos tienen el deber de respetar y conservar el patrimonio histórico canario y de reparar el daño que se les cauce a los mismos".

"las administraciones competentes asegurarán el mantenimiento y conservación de los bienes del patrimonio histórico canario, con independencia de su titularidad o régimen jurídico, garantizando que su gestión se produzca sin merma de su potencialidad y de modo compatible con la finalidad de protección, preservándolos para las futuras generaciones".

En las Islas Canarias se han incoado y declarados como Bienes de Interés Cultural (B.I.C) varios molinos tradicionales de viento harineros ubicados en algunas de las islas del archipiélago. Por orden cuantitativo, la isla de Fuerteventura tiene declarados 23 molinos, la isla

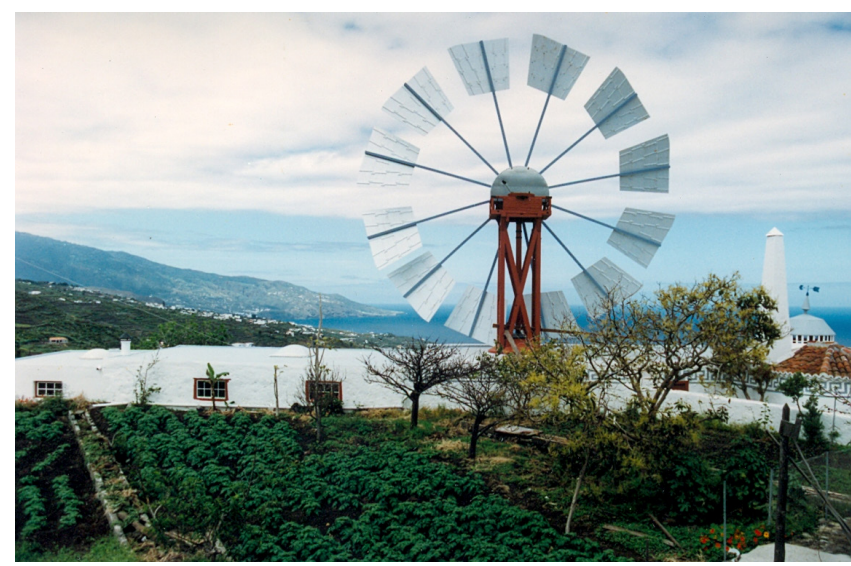

Figura 4.- Molino harinero "Sistema Ortega". TM Villa de Mazo. La Palma. Fotografía: Edvinia Barreto Cabrera

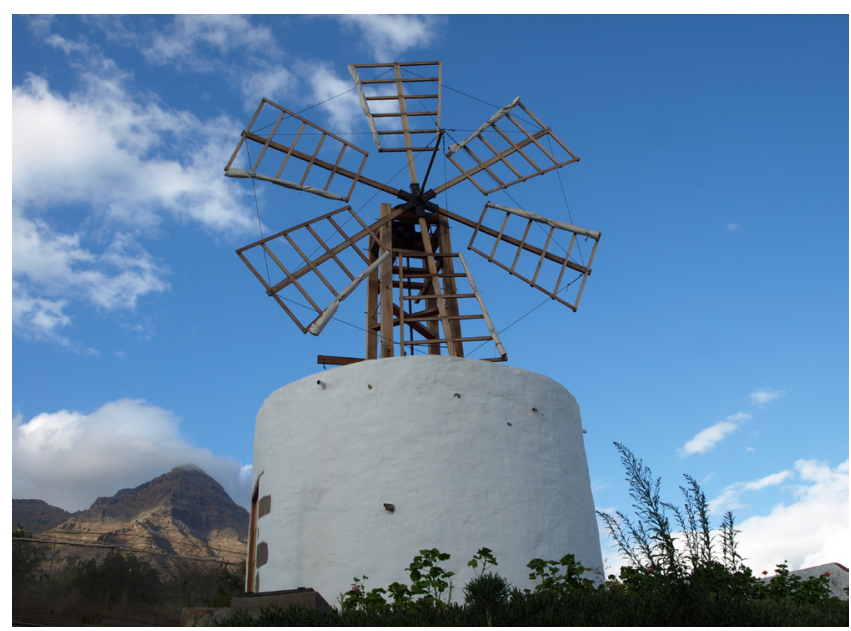

Figura 5.- Molino harinero "Sistema Romero". TM La Aldea. Gran Canaria. Fotografía: Emilio José Rodríguez Segura

de Tenerife tiene declarados 2 molinos, la isla de Gran Canaria tiene declarado 1 molino y en la isla de Lanzarote tiene declarado 1 molino de viento, todos ellos declarados como Bien de Interés Cultural (B.I.C) y principalmente atendiendo a dos categorías: Monumentos y/o Sitios Etnológicos. Los molinos de viento tradicionales incoados y declarados hasta este momento como B.I.C. responde principalmente a dos tipos, molinos de viento harineros "Tipo Torre" y molinos de viento harineros "Las Molinas". No se han incoado ni declarado hasta el momento como Bien de Interés Cultural los molinos de viento harineros del "Sistema Ortega" [figura 4] y del "Sistema Romero" [figura 5] existentes en algunas de las islas del archipiélago, ya que difieren mucho de los molinos de viento que se conocen en el resto de las islas Canarias y en el resto del territorio nacional.

\section{—La revitalización del medio rural}

El Patrimonio Histórico es perfectamente compatible con las necesidades económicas actuales y es un recurso potencial para nuestro futuro. En este sentido 
La ley 45/2007, de 13 de diciembre, para el Desarrollo Sostenible del Medio Rural pretende contribuir a que los ciudadanos que habitan en el medio rural puedan alcanzar un desarrollo suficiente y al mismo duradero. Para su aplicación la Ley exige la colaboración de todas las administraciones públicas, entre las cuales se encuentran las administraciones locales que deben de contribuir al desarrollo sostenible del mundo rural mediante la puesta en marcha de diversos programas de desarrollo rural.

Uno de los programas que se llevan a cabo para el desarrollo del medio rural es la agricultura ecológica, potenciando el consumo de productos locales de la tierra, apoyando la implantación de mercadillos de los propios agricultores donde se ofrecen varios productos rurales de calidad sin perder la identidad cultural, alimentos tradicionales y sanos que permitirían una revitalización del paisaje agrícola y de la economía en el medio rural de las islas del archipiélago. El aprovechamiento y el incremento de los productos agroalimentarios de los programas de desarrollo del medio rural han sido gracias a la mejora de la calidad, la modernización de las estructuras productivas y la diversificación con nuevos productos. La artesanía y los productos gastronómicos típicos benefician al medio rural ofreciendo a los ciudadanos de una comunidad como a los visitantes numerosos productos naturales, artesanales y turísticos con valores medioambientales.

Otro de los programas que se llevan a cabo es el relacionado con el turismo sostenible enfocado hacia el Patrimonio, la cultura, y la naturaleza, lo que supondría una contribución eficaz al desarrollo económico del medio rural. En la actualidad, el llamado turismo rural que incluye el ecoturismo, el agroturismo, el turismo arqueológico (histórico-cultural) y el turismo de aventura tienen un desarrollo importante, contribuyendo igualmente al desarrollo económico del medio rural. Estos cambios del turismo son debidos al cambio del perfil del turista quien posee cierto nivel cultural, educativo y mejores condiciones de vida. La creación de las denominadas rutas turísticas, que son itinerarios que permiten conocer de forma organizada un proceso productivo en el medio rural o urbano como expresión de una identidad cultural. La organización de distintas y diversas rutas como son las gastronómicas, artesanales, industriales, culturales y ambientales posibilitan la reactivación de la economía local, y siempre con la participación ciudadana fruto de la colaboración estrecha entre el sector público y el sector privado. La valorización y la recuperación de nuestro Patrimonio Histórico suponen un valor potencial para las necesidades actuales y futuras y para ello el apoyo a los oficios tradicionales mediante las escuelas taller resultan imprescindibles. La creación de un entorno sostenible en el medio rural con los recursos del lugar ligado con las alternativas de ocio compatibles con la preservación ambiental de turismo alternativo supone realizar actuaciones que impulsen el empleo en aras de garantizar la economía sostenible a partir de los recursos naturales, así como la protección del medio rural. De esta manera un desarrollo integral del medio rural impulsado por la economía local hará productiva la inversión en la recuperación de nuestro Patrimonio Histórico.

\section{—La revitalización de los molinos de viento}

Los molinos de viento tradicionales son unas construcciones que pertenecen a la arquitectura tradicional y están englobados en la categoría de la "arquitectura para el trabajo" relacionada con los procesos de producción y transformación. Una de las características más notables de la arquitectura tradicional es su sostenibilidad, ya que utiliza todos los recursos disponibles del entorno natural y del territorio donde se ubica. La reutilización de las edificaciones y de los materiales constructivos, de las herramientas y de las maquinarias facilitan la conservación y regeneración del territorio, porque al utilizar los materiales que necesita, permite sanear el entorno donde se ubican y nos permite entender la simbiosis tan natural que existe entre las edificaciones, el entorno, las actividades económicas y las maneras de habitar de una determinada sociedad en un medio bioclimático.

Ante la grave situación actual de desidia, abandono y ruina de gran cantidad de edificaciones pertenecientes a la arquitectura tradicional en el mundo rural, se propone que se apliquen nuevas formas de producción ecológica, adaptando para ello ciertas edificaciones e infraestructuras que, en muchos casos, han dejado de tener vigencia desde hace varias décadas, y que en la actualidad servirían para alojar determinados usos que pudieran tener un desarrollo sostenible y que se podrían incorporar a la sociedad actual. Por lo tanto, para poder recuperar con éxito la gran variedad de edificios históricos pertenecientes al Patrimonio arquitectónico tenemos que dotarlos de usos actuales que sean compatibles con estas construcciones, de no ser así, resultaría una acción en vano.

Los Museos y Centros de Interpretación son y han sido esenciales para la difusión educativa, ciudadana y turística, complementarios a la política y práctica de salvaguarda del patrimonio industrial nacional y regional. En estos años se han puesto de manifiesto ejemplos significativos donde se ha conciliado la exigencia de reutilización económica viable con la valoración cultural respetuosa. La escasa atención por parte de las administraciones públicas para la salvaguarda de este patrimonio, sus carencias en el marco legislativo, no son ajenas a una insuficiente sensibilización por parte de los agentes sociales hacia la valoración, conservación y protección de estos elementos denota, todavía, falta de influencia de las asociaciones de Defensa del Patrimonio Industrial en muchos lugares (Álvarez 2015)

En la actualidad, las máquinas eólicas se utilizan generalmente para producir energía eléctrica a través de los aerogeneradores, buscando incorporar a las energías renovables al sistema de la red eléctrica. Sin embargo, los tradicionales molinos de viento son elementos de la 
arquitectura tradicional que pertenecen al pasado debido a que han desaparecido los modos de vida a los que iban ligados.

Como alternativa a las diversas estrategias existentes destinadas a procurar la conservación de estas construcciones pertenecientes a la arquitectura tradicional se propone recuperar el funcionamiento de estos molinos de viento dotándoles de un nuevo uso, es decir, implantándoles una tecnología específica que les permita producir energía eléctrica a partir de la energía renovable del viento mediante el acoplamiento de un generador de baja potencia, iniciativa similar a la llevada a cabo por el Ayuntamiento de Campos, en Palma de Mallorca en el año 2000 (Cabrera 2009).

El Proyecto de Recuperación Patrimonial de Molins de Campos denominado MOLCAMP se ha realizado según los acuerdos entre el Ministerio de Medioambiente, el ajuntament de Campos, Nueva Gedisa (Grupo Endesa) y el IDAE (Instituto para la Diversificación y el Ahorro Energético del Ministerio de Fomento) en la zona ubicada entre la villa de Campos y la playa dEs Trenc. Este Proyecto permite acercar al hoy estos ingenios eólicos dotándolos de la tecnología actual para poder desempeñar su papel de aprovechamiento energético del viento para la producción de energía eléctrica resolviendo los problemas técnicos que ello conlleva, a la vez que mejorando su impacto visual, estético y medioambiental de la zona en la que están implementados (Pascual 2003)

Esta nueva propuesta posibilitaría dar respuesta a la inoperatividad actual de los diversos tipos de molinos de viento tradicionales, incorporándoles un nuevo uso y que es perfectamente compatible con estas construcciones pertenecientes a la arquitectura tradicional y al Patrimonio Industrial. Con la iniciativa planteada se podría recuperar lo que aún no se perdido de estas construcciones procedentes del patrimonio industrial tradicional canario, y que al mismo tiempo el nuevo uso es compatible con las necesidades sociales actuales en el interés creciente por obtener energía eléctrica a través de las energías limpias y renovables, en aras de disminuir las emisiones de $\mathrm{CO}_{2}$ a la atmósfera (Protocolo de Kioto, Japón). La energía eléctrica obtenida se puede utilizar para dar servicio a las edificaciones complementarias vinculadas a los molinos de viento como son los talleres y tiendas artesanales y/o para dar servicio al alumbrado público de calles, plazas, parques y jardines. Debido a que los molinos de viento tradicionales existentes en las islas Canarias llevan muchas décadas sin utilizarse $y$, por consiguiente, sin un mantenimiento adecuado, conlleva a que la mayor parte de los materiales constructivos de los mismos, mayoritariamente los elementos construidos en madera se encuentren deteriorados debido fundamentalmente a la erosión y al desgaste que han originado los agentes climatológicos.

Los elementos de los molinos de viento que se encuentran deteriorados deben de ser sustituidos por otros elementos de nueva factura, por lo que se tiene que tener en cuenta que las nuevas intervenciones y los nuevos elementos se deben de diferenciar de las originales (García; Iniesta; Lema 2008). Las diversas actuaciones a realizar en los distintos tipos de molinos de viento se plantearán caso a caso, por lo que en cuanto a la rehabilitación se refiere, tanto la sustitución de los elementos en estado ruinoso como la incorporación de nuevos elementos constructivos han de tener en cuenta la legislación vigente en materia de Patrimonio.

- Carta de Atenas para la restauración de monumentos históricos. 1931.

- Carta Internacional para la Conservación y Restauración de Monumentos y Sitios. ICOMOS. Venecia 1964.

- Convenio Europeo para la Protección del Patrimonio Arqueológico. Consejo de Europa. Londres. 1969. Resultado de la evolución de las políticas urbanísticas de los países europeos.

- Declaración de Ámsterdam que recoge los principios contenidos en la Carta Europea del Patrimonio Arquitectónico. Consejo de Europa. 1975.

- Informe Warburton de 1983. Consejo de Europa. Se hace eco de la disociación entre la tutela de monumentos históricos y la regulación del urbanismo en general. Se considera imprescindible proteger los monumentos y el entorno. Sus planteamientos son recogidos en la ley 16/1985 del PHE mediante la figura de los planes especiales.

- Convenio para la Salvaguarda del Patrimonio Arquitectónico de Europa. Consejo de Europa. Granada. 1985. Ratificado por España en 1989.

- Carta Internacional para la Conservación de Poblaciones y Áreas Urbanas Históricas. ICOMOS. Toledo 1987.

- Recomendación no R (98) 4 sobre medidas para promover la conservación integral de complejos históricos compuestos por propiedades muebles e inmuebles. Comité de Ministros del Consejo de Europa.

- Recomendación no R (91) 13 sobre la protección del Patrimonio Arquitectónico del siglo XX. Comité de Ministros del Consejo de Europa.

- Convenio Europeo sobre la protección del Patrimonio Arqueológico. Consejo de Europa. La Valetta, 1992. (Revisa el de 1969).

- Principios para la creación de archivos documentales de Monumentos, Conjuntos Arquitectónicos y Sitios Históricos y Artísticos. ICOMOS. Sofía. 1996.

- Recomendación 1486 (2000) sobre el patrimonio cultural marítimo y fluvial. Consejo de Europa.

- Carta de Cracovia, 2000.

- Convenio Europeo del Paisaje. Consejo de Europa. Florencia. 2000.

- Convención sobre el valor del patrimonio cultural para la sociedad. Consejo de Europa. Faro. 2005

- Ley 16/1985, de 25 de junio, del Patrimonio Histórico Español.

- Ley 4/1999, de 15 de marzo, de Patrimonio Histórico de Canarias

En líneas generales, se trataría fundamentalmente de consolidar las fábricas resistentes, los revestimientos interiores y exteriores de los edificios, así como los 
elementos de la maquinaria con la reparación y/o sustitución de los elementos deteriorados realizados en madera diferenciándolos de los originales, Art.12, Carta de Venecia 1964, “.......los elementos destinados a remplazar las partes inexistentes deben integrarse armoniosamente en el conjunto, distinguiéndose claramente de las originales a fin de que la restauración no falsifique el documento artístico o histórico"

\section{Discusión y resultados}

La dependencia energética exterior del archipiélago canario es cada vez mayor ya que se carecen en las islas de recursos energéticos convencionales y esto trae consigo riegos de carácter económicos, sociales y ecológicos para los habitantes de las islas. El sistema energético de las islas está basado en la combustión de los combustibles fósiles (petróleo y sus derivados) que se importan desde el exterior a través del transporte marítimo.

El mantenimiento de este sistema energético no resulta sostenible ya que se están agotando las reservas de dichos combustibles y esto origina grandes tensiones económicas entre los países industrializados por hacerse con dichas reservas para su explotación y además contribuyen al efecto invernadero, por lo tanto, el sistema energético de Canarias tendrá que evolucionar hacia el empleo de la energía primaria obtenida a partir de las energías renovables, que son aquellas que se producen de forma continua, son inagotables y se renuevan continuamente a diferencia de los combustibles fósiles. Durante los últimos años se ha fomentado la utilización de energías renovables en las islas que pueden limitar y/o sustituir en gran medida el empleo de las energías convencionales, reduciendo de esta forma la dependencia energética del exterior, favorecería el desarrollo de la industria local y mejoraría la imagen del archipiélago canario ante el turismo

Actualmente el consumo de energía crece sin parar en las islas debido fundamentalmente al incremento vertiginoso del sector del turístico siendo el único sector económico que goza de una cierta bonanza económica, sin embargo, el deterioro de la competitividad, la inexistencia de una planificación medioambiental, el envejecimiento de las infraestructuras turísticas, la gestión conservadora de los promotores y las exigencias de los turoperadores respecto al modelo territorial basado en el consumo intensivo del suelo y de la energía se erigen como amenazas para este sector económico en los años venideros. La tendencia actual del modelo turístico tendrá que evolucionar hacia la sustentabilidad, es decir, hacia un modelo basado en un desarrollo sostenible bajo las premisas de conservación y mejora de los recursos naturales, evitando el consumo intensivo de suelo, agua y de la energía. Ante la amenaza que representa el cambio climático para toda la humanidad, la comunidad internacional se reunió en la ciudad de Cancún en 2010 para adoptar una serie de mejoras y acuerdos con la mente en las generaciones futuras, de entre estas iniciativas destacan el compromiso de reducir las emisiones de $\mathrm{CO}_{2}$ a la atmósfera, evitar la sobreexplotación de los recursos naturales. La sostenibilidad energética debe referirse, entre otras cuestiones, a la eficiencia en el consumo energético referido a la iluminación artificial, la maquinaria, los motores, etc. y la incorporación de las energías renovables y limpias al sector energético (ARMAS; SANTANA; CABRERA 2015).

En Canarias, el consumo energético dedicado al transporte representa aproximadamente el $70 \%$ de la demanda de energía final. La energía primaria se obtiene utilizando los combustibles fósiles importados del exterior, algo que sabemos que tiene que cambiar con el fin de reducir la contaminación y las consiguientes emisiones de $\mathrm{CO}_{2}$ a la atmósfera. Con respecto a la energía secundaria o final, el sector del transporte en sus tres modalidades (marítimo, aéreo y terrestre), representa el de mayor consumo directo y es a este sector al que se le destina la mayor parte del consumo de los combustibles fósiles derivados del petróleo (fuel-oil, gasolinas, queroseno, etc.), y el resto, se utiliza para la producción de energía eléctrica, que es generada principalmente por las centrales térmicas para dar suministro de energía en la edificación (aire acondicionado y calefacción, agua caliente sanitaria, electrodomésticos e iluminación) corresponde a una parte del porcentaje total consumido al compararlo el consumo del sector de transporte. Las limitaciones del suministro, del consumo y del coste económico en la generación de la energía tienen un peso determinante en las posibilidades de desarrollo económico y competitividad en las Islas Canarias (Cabrera; Santana; Armas 2016).

La Unión Europea (UE) ha dispuesto una serie de directivas que permitirán cumplir con del Protocolo de Kioto por el cual todos los países miembros deben de cumplir tres objetivos obligatorios para el año 2020: La reducción del $20 \%$ de las emisiones de gases de efecto invernadero, la mejora de la eficiencia energética en un $20 \%$ y la incorporación de las energías renovables al 20\% del consumo energético. Tendremos que buscar soluciones a la contaminación industrial y/o ambiental de nuestro planeta derivado de quema de los combustibles fósiles y contaminantes para obtener la energía. Las tecnologías para producir energías alternativas renovables a los combustibles fósiles, son necesarias no solo por motivos medioambientales, sino por el agotamiento de las fuentes convencionales.

En cualquier caso, se empieza a tomar conciencia de que los beneficios de contaminar menos superan a los costos, por lo que sería interesante crear una conciencia social en el que el coste ecológico de la producción en la obtención de la energía no penalice a los consumidores por el mayor valor económico de la misma, sino al contrario, que lo asuman como un bien ecológico frente a otros productos energéticos a priori más económicos, pero producidos con un coste medioambiental mayor. 
Un molino de viento es una máquina que convierte una determinada forma de energía procedente de su fuerza motora, el viento, el agua, un combustible, etc., en energía mecánica, capaz de mover un mecanismo que produce un trabajo útil para el hombre. Aunque el término molino está relacionado con "moler", se ha aplicado esta denominación a toda máquina cuya energía se capta con un dispositivo giratorio, aunque su objetivo último no sea moler grano (Valera 2010)

Por lo tanto, la estrategia planteada de recuperar los molinos de viento tradicionales mediante su conversión en aerogeneradores de baja potencia con la finalidad de obtener energía eléctrica mediante la energía renovable del viento se alinea con los objetivos indicados anteriormente. Aunque la generación de energía eléctrica que se podría obtener a partir de los tradicionales molinos de viento no resulte del todo significativa respecto las cantidades de energía que obtienen de los actuales aerogeneradores agrupados mayoritariamente en parques eólicos, los molinos de viento tradicionales pueden desempeñar una función pública en cuanto a la producción de energía eléctrica, ya que dicha energía podría utilizarse para dar servicio a la iluminación del espacio público como pueden ser determinados tramos de la red viaria, plazas urbanas o parques públicos, sectores donde el suministro energético no sea elevado respecto a las prestaciones de generación de energía eléctrica que podrían generar estos edificios singulares de la arquitectura tradicional pertenecientes al Patrimonio Industrial.

Las potencias máximas que se puede obtener de los tradicionales molinos de viento para generar energía eléctrica depende fundamentalmente de dos parámetros, de un lado, el diámetro del rotor de aspas y de otro lado, la velocidad del viento (Cabrera 2017). La energía eléctrica que se podría obtener se indica mediante el siguiente dibujo comparativo [figura 6]
Según el libro Verde: Iluminemos el Futuro (Comisión Europea 12-12-2011), en la UE la proporción que representa la iluminación interior en el consumo total de electricidad varía considerablemente en función del tipo de edificios, sin embargo, respecto a la climatización artificial (calefacción y aire acondicionado) representa el 70\% del consumo de electricidad mientras que la iluminación y el resto electrodomésticos representan un 30\% del total, correspondiendo a la iluminación el $14 \%$ del consumo de electricidad en la UE, y correspondiendo al 1.35\% el consumo del alumbrado exterior y el $0.4 \%$ el consumo en autovías y carreteras $^{[2]}$. El alumbrado público facilita la orientación y aumenta la seguridad de las personas al tiempo que se emplea cada vez con mayor frecuencia el efecto de la iluminación para crear ambientes. Los requisitos que debe cumplir un sistema de alumbrado son muy exigentes ya que deben operar bajo cualquier condición meteorológica, cumplir las normas de iluminación y consumir la menor cantidad de energía posible siendo lo más adecuado optimizar las horas de funcionamiento de las luminarias y reducir transitoriamente su potencia, además de emplear lámparas de alto rendimiento (LED, lámparas de vapor metálico y lámparas de vapor de sodio) que reducen en gran medida los costes de energía.

Otra estrategia sería adaptar el nivel de iluminación a la densidad de la red viaria referente al tráfico durante la noche, siendo una medida eficaz la de bajar la iluminancia hasta el $50 \%$ durante las horas de menos frecuencia de uso. No obstante, parece oportuno señalar que se debe de realizar una serie de cambios para conseguir un ahorro significativo en la iluminación pública, ya que esta permanece encendida durante mucho tiempo, por lo tanto se propone lo siguiente:

- Adecuar los niveles de iluminación eliminando lámparas o reducir la potencia de los puntos de luz y/o cambiar las luminarias actuales.

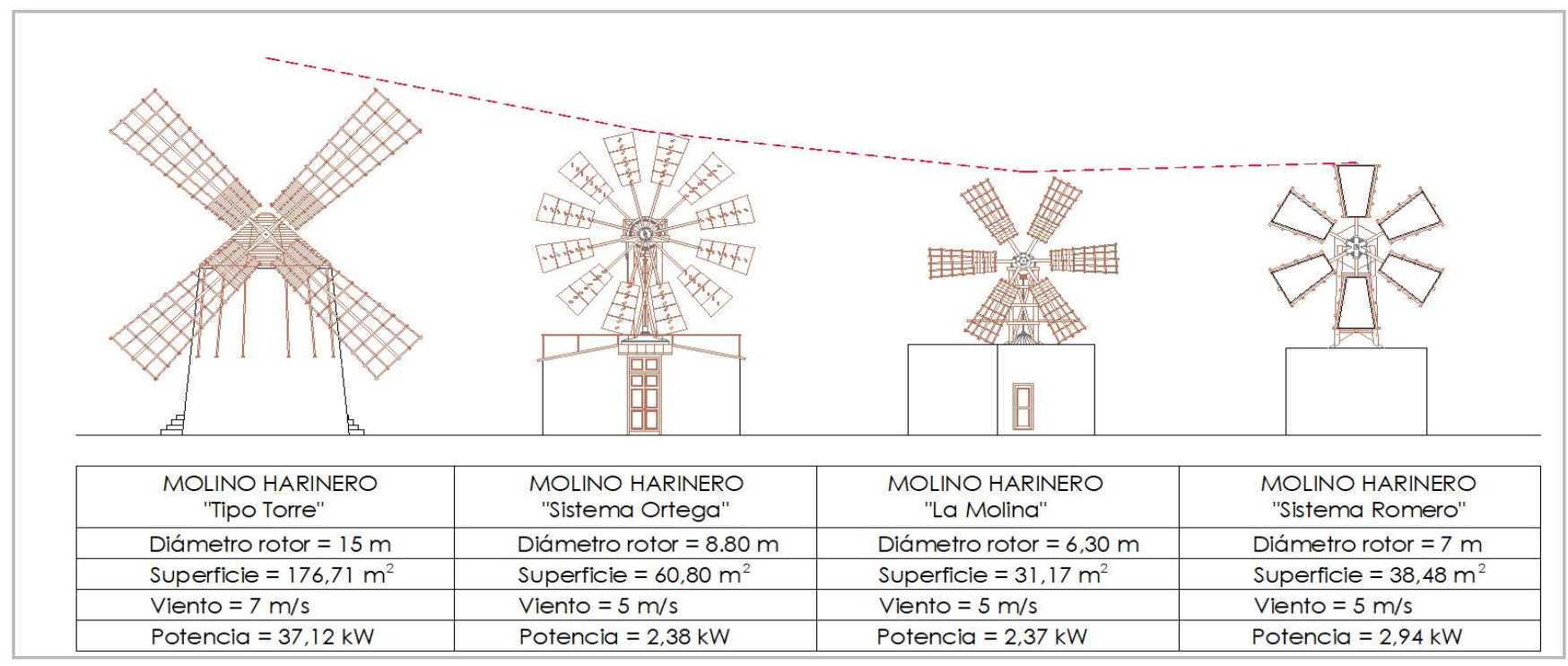

Figura 6.- Producción de energía eléctrica de molinos tradicionales de viento. Elaboración autor 
- Sustitución de luminarias de baja eficiencia energética, obligadas por el conjunto de leyes y ordenanzas puestas en marcha para disminuir la contaminación lumínica en alumbrado que anula la visión del cielo estrellado.

Es en este último concepto del alumbrado público donde la iniciativa de producir energía eléctrica a partir de los molinos de viento tradicionales tendría un mayor sentido debido al escaso porcentaje de suministro energético en relación a otros conceptos, ya que el sector de la iluminación pública es uno de los sectores en los que es posible obtener un mayor ahorro energético con relativa facilidad.

\section{Conclusiones}

Los tradicionales molinos de viento existentes en el archipiélago canario son unas edificaciones singulares que pertenecen a la arquitectura tradicional, y que están englobados en la categoría de la "arquitectura para el trabajo" relacionada con los procesos de producción y transformación, por lo que son elementos del Patrimonio Industrial. Intentar recuperar parte de la arquitectura tradicional sin proponer usos alternativos en los edificios es una acción en vano, ya que no todas las construcciones de la arquitectura tradicional pueden convertirse en museos y/o Centros de Interpretación. Ante la situación actual de desidia y de abandono por parte de la sociedad actual que permite que gran cantidad de estas edificaciones se encuentren en ruinas favoreciendo su desaparición, se propone actualizar el uso de los molinos de viento tradicionales de tal manera que podamos rescatarlos del olvido y podamos convertirlos en aerogeneradores de baja potencia, con la intención de generar energía eléctrica a partir de la energía limpia y renovable del viento. Actualmente es posible la incorporación con garantías de algunos mecanismos de los actuales aerogeneradores para con los molinos tradicionales sin que se produzcan distorsiones significativas en estas construcciones de la arquitectura tradicional.

Actualizar los edificios de la arquitectura tradicional con usos alternativos que sean compatibles para la sociedad actual supondría que los proyectos de rehabilitación que permitan la revitalización de los edificios históricos se tienen que ajustar a lo indicado en la legislación patrimonial $y$, además, deben de cumplir con las diferentes normativas técnicas de ámbito nacional y de obligado cumplimento principalmente referente a:

- Reforzar la estructura de los edificios ante las nuevas cargas (nuevos usos).

- Diseño de elementos que favorezcan la accesibilidad en los edificios.

- Diseño de las medidas de seguridad contra incendios.

La propuesta de cambio de uso (producir energía eléctrica) en los molinos de viento tradicionales posibilitaría dar respuesta a la inoperatividad actual en estos elementos del Patrimonio Industrial, permitiéndonos poder recuperar lo que aún no se perdido de estas construcciones procedentes de la arquitectura tradicional y que al mismo tiempo es compatible con las necesidades sociales actuales en el interés creciente por obtener energía eléctrica a través de las energías limpias y renovables, en aras de disminuir la emisiones de $\mathrm{CO}_{2}$ a la atmósfera (Protocolo de Kioto 2020). Los molinos de viento tradicionales pueden desempeñar una función pública en cuanto a la producción de energía eléctrica, ya que dicha energía podría utilizarse para dar suministro a la iluminación del espacio público (red viaria, parques y jardines) debido al escaso porcentaje de suministro energético en relación a otros conceptos, y que, además, es un sector donde se podría obtener una mayor eficiencia energética. La incorporación de este nuevo uso a los molinos de viento es compatible para la sociedad actual y posibilitaría planificar mantenimientos periódicos tanto de los edificios, los rotores de aspas y en la maquinaria, por lo tanto, se podría garantizar la conservación de los mismos para las generaciones futuras. En este sentido, cabe destacar la experiencia surgida en un paraje de los Países Bajos, concretamente en Kinderdijk, conjunto de pólder (tierras ganadas a los humedales y protegidas por diques) y declarada hace dos décadas como patrimonio de la humanidad por la UNESCO, donde se sitúan diecinueve molinos de viento tradicionales que bombean agua a las lagunas de almacenaje y de ahí a los ríos cercanos, donde los molinos de viento construidos entre los siglos XVII y XVIII continúan realizando un trabajo en concreto y útil para la sociedad actual, lo que facilita su conservación para generaciones futuras a la vez que se convierten en un recurso económico y atractivo para el turismo de la sociedad actual.

En definitiva, estamos obligados por Ley a conservar los elementos materiales e inmateriales más importantes de nuestro Patrimonio Cultural para el uso, el estudio y el disfrute de los mismos tanto para nuestra sociedad como para las generaciones futuras, por lo tanto, el conocimiento, la creatividad y la diversidad de soluciones a plantear en que hacer con el Patrimonio hoy en día deben de ser recursos inagotables para la sociedad actual, con la finalidad de trasladar en las mejores condiciones posibles los bienes patrimoniales que hemos heredado de nuestros antecesores a las generaciones venideras.

\section{Notas}

[1] Plan Nacional de Arquitectura Tradicional.

[2] Guía sobre tecnología Led del alumbrado. Fundación de la Energía de la Comunidad de Madrid.

\section{Bibliografía}

ÁLVAREZ ARECES, M.A (2015). "Patrimonio y turismo en lugares de tradición industrial". Territorios con referencias y sentido del lugar. Jornadas de Patrimonio y Turismo, convergencias y propuestas. Madrid: IPCE. 
ARMAS CABRERA, M.E, SANTANA RODRÍGUEZ, R.J, CABRERA GARCÍA, V.M, (2015) “Turismo de sol y playa en la zona del atlántico norteafricano. Los ejemplos de Maspalomas Costa Canaria y de Agadir". I Foro Internacional de Emprendimiento e Investigación en Turismo. Las Palmas de Gran Canaria, 69-80.

BENITODELPOZO,P,CALDERÓNCALDERÓN, B, RUIZVALDEPEÑAS, H.P (2016). “La gestión territorial del patrimonio industrial en Castilla y León (España): Fábricas y paisajes". Investigaciones geográficas, Boletín del Instituto de Geografía, UNAM No 90, 136-154.

CABRERA GARCÍA, V.M, ARMASCABRERA, M.E, SANTANA RODRÍGUEZ, R.J, (2016) "La Eficiencia Energética de las Edificaciones Turísticas en el Archipiélago Canario". IV Foro Internacional de Turismo Maspalomas Costa Canaria. San Bartolomé de Tirajana, Gran Canaria, 55-64.

CABRERA GARCÍA, V. M. (2017). "Aprovechamiento energético de los molinos de viento tradicionales de las islas Canarias". Revista Técnica Industrial № 317, agosto 2017, 58-66.

CABRERA GARCÍA, V. M. (2010). "Molinos de viento en las Islas Canarias". Tenerife. Editorial: Ediciones Idea S.A. Colección: Territorio Canario.

CABRERA GARCÍA, V. M. (2009). "La Arquitectura del Viento en Canarias. Los molinos de viento. Clasificación, funcionalidad y aspectos constructivos". Tesis Doctoral, Universidad de Las Palmas de Gran Canaria.

CASTRO GARCÍA, J, ROJASSOLA, J.I, CARRANZA CAÑADAS, M.P (2013). "Caracterización tecnológica de los molinos de viento mediterráneos Españoles". Medellín. DYNA. Volumen: 80, №.177, 22-30.

Consejería de Economía y Hacienda de la Comunidad Autónoma de Madrid. Fundación de la Energía de la Comunidad de Madrid (2015). “Guía sobre tecnología led del alumbrado”. BOCM, 7.

DE DECKER, K. (2011). http://www.es.lowtechmagazine. com/2011/08/pasado-y-futuro-de-los-molinos-de-vientoindustriales.html [Consulta 22.04.2018].

ESPAÑA. LEY 16/1985, de 25 de junio, de PATRIMONIO HISTÓRICO ESPAÑOL. Gobierno de España.

ESPAÑA. LEY 4/1999, de 15 de marzo, de PATRIMONIO HISTÓRICO DE CANARIAS, modificada por La LEY 11/2002, de 21 de Noviembre. Gobierno de Canarias.

ESPAÑA. LEY 45/2007, de 13 de diciembre para el Desarrollo Sostenible del Medio Rural.

ESPAÑA. Ministerio de Educación, Cultura y Deporte (2015). Plan Nacional de Arquitectura Tradicional.

GARCÍA SIMÓ, I, INIESTA SANMARTÍN, A, LEMA CAMPILLO, A (2008). "Molinos de viento en la Región de Murcia". Murcia. Edita: Comunidad Autónoma de la Región de Murcia. Consejería de Cultura, Juventud y Deportes. Dirección General de Bellas Artes y Bienes Culturales y Servicio de Patrimonio Histórico.
GONZALEZ DURAN, S. (2017). "Urban and industrial tourism in the "post-industrial periphery: Imaginaries and narratives of the inhabitants of the Left Bank of the river Nervion in metropolitan Bilbao." Scripta nova-revista electrónica de geografía y ciencias sociales. Volumen: 21, № 572.

GRANDE ALVAREZ, N. (2017). "Musealization of Industrial Heritage" .Two models of participation in the province of Huelva: the House of the General Manager in Valverde del Camino and Tide Mill "El Pintado" in Ayamonte". PASOS. Revista de Turismo Y Patrimonio Cultural. Volumen: 15, № 3, 659-672.

LALANDA ORDÓÑEZ, R, GÓMEZ-ELVIRA GONZÁLEZ, M.A, CARMENADO, I (2010). "Instrumento de apoyo para la puesta en valor y gestión sostenible de antiguos edificios de valor históricocultural en España: Marco ordenador, objetivos e indicadores". Comunicación. XIV International Congress on project engineering. Madrid.

PARDO ABAD, C. J. (2004). "La reutilización del patrimonio industrial como recurso turístico. Aproximación geográfica al turismo industrial". Treballs de la Societat Catalana de Geografía № $57,7-32$.

PASCUAL TORTELLÁ, J. (2003). "Reconversión de molinos de viento tradicionales de extracción de agua de Campos, Mallorca, para la producción de energía eléctrica". IV Congreso Internacional de Molinología. Volumen: 2, 2005, 369-380.

ROJAS SOLA, J.I, GÓMEZ BUENO, M.C, CASTRO GARCÍA, M (2013). "Molinos de viento en Andalucía: Nuevas herramientas para su puesta en valor". Boletín de la Asociación de Geógrafos Españoles NN 62, 403-227.

VALERA MARTÍNEZ-SANTOS, F. (2010). "Principios físicos y tecnología del molino de viento". https://www.campodecriptana. info/.../Fisica-y-tecnologia-del-molino-de-viento.pdf. [Consulta 17.01.2017] 


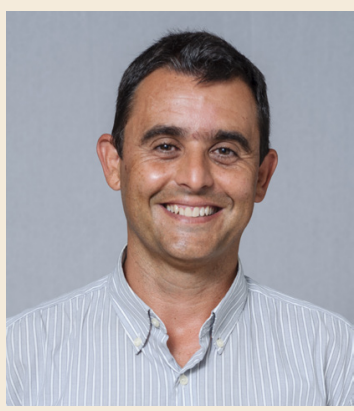

\section{Víctor Manuel Cabrera García}

victormanuel.cabrera@universidadeuropea.es

Universidad Europea de Canarias

Doctor Arquitecto en Restauración y Rehabilitación Arquitectónica por la Universidad de Las Palmas de Gran Canaria. Arquitecto con las especialidades de Edificación y Urbanismo por la Universidad de Las Palmas de Gran Canaria. Técnico Superior en Sistemas Electrotécnicos y Automatizados por el I.E.S Felo Monzón en Las Palmas de Gran Canaria. Arquitecto especialista en diseño, cálculo y ejecución de estructuras en la edificación. Desde el año 2014 es profesor del Grado de Fundamentos de la Arquitectura de la Escuela de Arquitectura de la Universidad Europea de Canarias, y profesor colaborador en "Venia Docendi" en el Grado de Arquitectura de la Escuela de Arquitectura de la Universidad de Las Palmas de Gran Canaria. También es Investigador Principal del Grupo de Investigación "Arquitectura y Desarrollo Turismo Sostenible" de la Universidad Europea de Canarias. Investigador adscrito al Grupo de Investigación Emergente de la Universidad Europea de Valencia con el nombre de "APP (Arquitectura-Pensamiento-Procesos)", coordinado por el Investigador Principal Dr. D. José Fernández-Llevez Muñoz. Investigador de la Asociación para la Conservación y Estudio de los Molinos "ACEM", entidad sin ánimo de lucro interesada en el estudio de los molinos y su entorno, los edificios, los elementos y los mecanismos que utilicen para su funcionamiento las distintas energías tradicionales.

\section{Artículo enviado el 20/12/2017 \\ Artículo aceptado el 07/05/2018}

